\title{
Kefiran Production by Lactobacillus kefiranofaciens under the Culture Conditions Established by Mimicking the Existence and Activities of Yeast in Kefir Grains
}

\author{
Masayuki TANIGUCHI, Masashi NOMURA, Takahiro ITAYA and Takaaki TANAKA \\ Department of Materials Science and Technology, Niigata University, 2-8050, Ikarashi, Niigata 950-2181, Japan
}

Received July 25, 2001; Accepted September 19, 2001

\begin{abstract}
Kefiran production by Lactobacillus kefiranofaciens alone under the culture conditions established by mimicking the presence and activities of yeast cells in kefir grains was investigated. When the pH of the culture broth was controlled at pH 5.5 by adding a $4 \mathrm{~N}-\mathrm{NaOH}$ solution during cultivation, cell growth and kefiran production were stimulated as compared with those in the cultivation without $\mathrm{pH}$ control. The addition of $5 \mathrm{~g} / \mathrm{l}$ of yeast extract to the medium was essential for kefiran production. By sparging a mixed gas of $\mathrm{N}_{2}$ and $\mathrm{CO}_{2}$ at a volume ratio of 9:1 into the culture broth at $0.3 \mathrm{vvm}$ throughout the cultivation, a slight increase in the amount $(670 \mathrm{mg} / l)$ of kefiran produced was observed as compared with that $(650 \mathrm{mg} / l)$ in the cultivation without aeration. When $10 \mathrm{~g} / l \mathrm{or} 20 \mathrm{~g} / l$ of ethanol was added to the medium containing $50 \mathrm{~g} / l$ of lactose, the kefiran concentrations were about $930 \mathrm{mg} / l \mathrm{or} 840 \mathrm{mg} / \mathrm{l}$ at 8 days, respectively. The maximum concentration of kefiran obtained was about $1040 \mathrm{mg} / l$ at 10 days, when $10 \mathrm{~g} / l$ of ethanol was added to the medium containing $75 \mathrm{~g} / l$ of lactose. These results showed that under the culture conditions established by mimicking the actions of yeast cells on $\mathbf{L}$. kefiranofaciens in kefir grains the amount of kefiran produced was enhanced even when only the lactic acid bacterium was used.
\end{abstract}

Keywords: kefiran, polysaccharide, Lactobacillus kefiranofaciens, lactic acid bacteria, co-culture with yeast

Lactic acid bacteria which are food-grade organisms possessing the GRAS (generally recognized as safe) status, are widely used in the food industry not only for lactic acid production but also for the formation of minor food components suitable for structure, flavor, and preservation. Several lactic acid bacteria are known to produce extracellular polysaccharides which contribute to the rheology and texture of fermented milk (Crescenzi, 1995). Polysaccharides produced by these bacteria also provide a source of stabilizing, viscosifying, emulsifying, gelling, and water binding reagents for use as natural food additives (Mukai et al., 1991), which may be an alternative to texturizing agents of plant and animal origins.

Kefiran, a water-soluble polysaccharide, is produced in kefir grains which consist of a complex population of lactic acid bacteria and yeasts firmly embedded (Tamime \& Robinson, 1988). The presence of yeasts results in the production of ethanol and also of carbon dioxide $\left(\mathrm{CO}_{2}\right)$ which gives an effervescent character to these products. Kefiran, which contains approximately equal amounts of glucose and galactose, is produced by Lactobacillus kefiranofaciens (Toba et al., 1986). The chemical structure and properties of kefiran extracted from kefir grains were investigated by Kooiman (1968), Mukai et al. (1990), and Micheli et al. (1999). The antitumor activity of kefiran was reported by Shiomi et al. (1982). To utilize kefiran in many applied fields such as food, cosmetic and pharmaceutical industries, it may be necessary to produce a large quantity of the polysaccharide by $L$. kefiranofaciens because extraction of kefiran from kefir grains cultured in milk is complicated and the yield is fairly low.

A homofermentative bacterium, L. kefiranofaciens which was

E-mail: mtanig@eng.niigata-u.ac.jp isolated from kefir grains by Toba et al. (1986) produced kefiran only in medium containing expensive wine, and kefiran was obtained at a low concentration $(80 \mathrm{mg} / l)$ in the culture. Yokoi et al. (1990) isolated a new kefiran-producing homofermentative bacterium, Lactobacillus sp. KPB-167B from kefir grains with a newly developed milk whey medium without wine, and examined the optimum culture conditions for production of kefiran by the lactic acid bacterium using MRS medium containing lactose (Yokoi \& Watanabe, 1992). Recently, Mitsue et al. (1998) obtained a high kefiran-producing strain (L. kefiranofaciens KF-75) suitable for industrial production of kefiran by repeated UV irradiation. They found that using the strain KF-75 higher kefiran productivity was successfully obtained by optimizing the medium and culture conditions in a 50- $l$ jar fermentor (Mitsue et al., 1999). In particular, they reported that the highest kefiran productivity could be achieved in a co-culture of the strain KF-75 and Torulaspora delbrueckii, one of the yeast strains existing in kefir grains (Mitsue et al., 1999).

We reported on not only the high concentration cultivation of several lactic acid bacterial cells using the fed-batch fermentation system involving a bioreactor with a microfiltration module (Taniguchi et al., 1987, 1988), but also the production of the useful substances, superoxide dismutase and an antibiotic polypeptide (nisin), by lactic acid bacteria (Taniguchi et al., 1989, 1994). We have given much attention to efficient production of kefiran by a co-culture of $L$. kefiranofaciens with yeast strains in kefir grains. Based on the results reported by Mitsue et al. (1999), we attempted kefiran production using a combination of L. kefiranofaciens and some yeast strains. Unfortunately, we were unable to obtain satisfactory results under the co-culture conditions. Accordingly, we focused on the influence of the co-existence of 
yeast on kefiran production by L. kefiranofaciens and identified this influence into factors resulting from the presence and activities of yeast strains. Thus, in the present paper, we investigated the effects of addition of yeast extract and ethanol, and the aeration of $\mathrm{CO}_{2}$ on kefiran production by L. kefiranofaciens.

\section{Materials and Methods}

Microorganisms The kefiran-producing lactic acid bacterial strains used in this study were Lactobacillus kefiranofaciens JCM 6985 and JCM 7446, and Lactobacillus kefir JCM 5818 and KL-3. For co-cultures with lactic acid bacteria, Saccharomyces cerevisiae IFO 0216, Candida kefyr IFO 10287 and Torulaspora delbrueckii IFO 1626 were used as yeast strains isolated from kefir grain.

Media and culture conditions Lactic acid bacteria were cultivated usually at $30^{\circ} \mathrm{C}$ in a modified MRS medium containing 5-10\% lactose, 1\% Polypepton (Nippon Seiyaku Co., Tokyo), $1 \%$ meat extract (Kyokuto Seiyaku Co., Tokyo), 0.5\% yeast extract (Oriental Yeast Co., Osaka), $0.5 \%$ sodium acetate, $0.2 \%$ ammonium citrate, $0.2 \% \mathrm{~K}_{2} \mathrm{HPO}_{4}, 0.2 \% \mathrm{MgSO}_{4} \cdot 7 \mathrm{H}_{2} \mathrm{O}$, and $0.05 \% \mathrm{MnSO}_{4} \cdot 5 \mathrm{H}_{2} \mathrm{O}$. The initial $\mathrm{pH}$ of the medium was adjusted to 5.5 unless otherwise noted. The medium was sterilized by autoclaving at $121^{\circ} \mathrm{C}$ for $15 \mathrm{~min}$. Lactic acid bacterial and yeast cells were precultured statically at $30^{\circ} \mathrm{C}$ in test tubes containing $10 \mathrm{ml}$ of the medium. The precultured cells were separated by centrifugation at $17,000 \times \mathrm{g}$ for $10 \mathrm{~min}$ and then inoculated into a fresh medium at an initial turbidity of about 0.5 at $660 \mathrm{~nm}$.

Lactic acid bacteria were cultivated batchwise in $500 \mathrm{ml}$ screw-capped medium bottles or a bioreactor (TBR-2, Sakura Seiki Co., Tokyo) with a working volume of $700 \mathrm{ml}$. Medium bottles were used for selecting a high kefiran-producing strain, and for examining the effects of basal culture conditions (carbon source, initial $\mathrm{pH}$, temperature, etc.) on cell growth and kefiran production. When the bioreactor was used, the $\mathrm{pH}$ of the medium was maintained at 5.5 by adding a $4 \mathrm{~N}-\mathrm{NaOH}$ solution with a peristaltic pump connected to a $\mathrm{pH}$ controller (FC-10, Tokyo Rikakikai Co., Tokyo). Since the presence of yeast in kefir grains results in the production of $\mathrm{CO}_{2}$ and ethanol, a mixed gas of $\mathrm{N}_{2}$ and $\mathrm{CO}_{2}$ at a volume ratio of 9:1 or 5:5 was sparged at $0.3 \mathrm{vvm}$ throughout the fermentation or/and 1 or $2 \%$ ethanol was added to the medium described above to evaluate the effects on kefiran production. The agitation rate was usually adjusted to $100 \mathrm{rpm}$.

Preparation and determination of kefiran Extracellular kefiran was recovered from the culture supernatant and determined. After centrifuging the culture broth at $17,000 \times \mathrm{g}$ for 10 min, kefiran in the supernatant obtained was precipitated by addition of an equal volume of cold ethanol. The resulting precipitate was collected by centrifugation at $17,000 \times \mathrm{g}$ for $10 \mathrm{~min}$ and dissolved with distilled water in $10 \%$ volume of a corresponding supernatant. The same procedure was repeated three times to purify the polysaccharides. The amount of kefiran was measured as total sugar according to the phenol-sulfuric acid method (Dubois et al., 1956).

Other analytical methods The cell concentration was determined by measuring the turbidity at $660 \mathrm{~nm}$. The number of bacterial and yeast cells was counted under a microscope using a hemocytometer. The supernatant obtained by centrifugation as described above was analyzed for determination of lactose, glu- cose, galactose, sucrose, and lactic acid concentrations. Lactose, glucose, galactose, and sucrose were determined by HPLC with a Shim-Pack CLC-101C column and a refractive index detector (RID-6A; both instruments from Shimadzu Seisakusho Co., Kyoto). Distilled water was used as the mobile phase at a flow rate of $1.0 \mathrm{ml} / \mathrm{min}$ at $80^{\circ} \mathrm{C}$. Lactic acid concentration was measured using an HPLC system for analysis of organic acids; the system was equipped with a Shim-Pack SCR 102H column and a conductivity detector (CCD-6A; both from Shimadzu Seisakusho Co.). Three $\mathrm{mM}$ of Bis-Tris solution containing $3 \mathrm{~mm} p$ toluenesulfuric acid and $100 \mu \mathrm{M}$ EDTA was used as the mobile phase at a flow rate of $0.8 \mathrm{ml} / \mathrm{min}$ at $40^{\circ} \mathrm{C}$.

\section{Results and Discussion}

Kefiran production using medium bottles To compare the amount of kefiran produced, four lactic acid bacterial strains isolated from kefir grains were cultivated using medium bottles containing the modified MRS medium with $50 \mathrm{~g} / \mathrm{l}$ of lactose at $30^{\circ} \mathrm{C}$ for 7 days. The initial $\mathrm{pH}$ of the medium was adjusted to 5.5. L kefir JCM 5818 and KL-3 grew rapidly on the first day. Although the cell growth of both strains almost stopped after 1 day, lactose was gradually consumed and lactic acid continued to accumulate in the culture broth with gradual decrease in $\mathrm{pH}$. The growth rates of the two L. kefiranofaciens strains were slower than those with L. kefir and the amounts of lactic acid produced were lower than those by L. kefir. However, the final kefiran concentrations were $200-250 \mathrm{mg} / \mathrm{l}$ and $400-500 \mathrm{mg} / \mathrm{l}$ for L. kefiranofaciens JCM 7446 and JCM 6985, respectively. These values were higher than the concentrations $(100-120 \mathrm{mg} / l)$ of kefiran produced by the two strains of L. kefir. L. kefiranofaciens JCM 6985 was selected as one of the most suitable strains for kefiran production and cultivated using glucose, galactose, lactose, sucrose or a mixed sugar of glucose and galactose as a carbon source under the culture conditions described above. Using $50 \mathrm{~g} / \mathrm{l}$ of lactose as a carbon source, the highest kefiran yield (about $500 \mathrm{mg} / \mathrm{l}$ ) was obtained at a cultivation time of 9 days. Moreover, the optimum conditions for initial $\mathrm{pH}$ and temperature were examined in the modified MRS medium containing $50 \mathrm{~g} / \mathrm{l}$ of lactose. The initial $\mathrm{pH}$ was adjusted to $4.5,5.5$, and 6.5 at $30^{\circ} \mathrm{C}$. The highest quantity of kefiran was obtained at the initial $\mathrm{pH}$ of 5.5 . When cultivations were carried out at $25^{\circ} \mathrm{C}, 30^{\circ} \mathrm{C}$, and $37^{\circ} \mathrm{C}$ under an initial $\mathrm{pH}$ of 5.5 , the highest yield of kefiran was achieved at $30^{\circ} \mathrm{C}$. Therefore, the subsequent cultivations were done using lactose as a carbon source under the conditions of initial $\mathrm{pH}$ of 5.5 and temperature of $30^{\circ} \mathrm{C}$.

Effect of addition of yeast cells Based on the results reported by Mitsue et al. (1999), the effect of addition of yeast cells on kefiran production by L. kefiranofaciens JCM 6985 was investigated. In the co-culture, L. kefiranofaciens and S. cerevisiae, C. kefyr, or T. delbrueckii at a concentration of $5 \times 10^{5}$ cells $/ \mathrm{ml}$ and $1 \times 10^{5}$ cells $/ \mathrm{ml}$, respectively, were inoculated simultaneously. Unfortunately, little or no positive effect of the addition of yeast cells on kefiran production by L. kefiranofaciens was observed. Similar results were obtained even in the co-cultures where the yeast cells at different concentrations $\left(1 \times 10^{3} \sim 1 \times 10^{5}\right.$ cells $\left./ \mathrm{ml}\right)$ were added to the culture containing $5 \times 10^{5}$ cells $/ \mathrm{ml}$ of $L$. kefiranofaciens and/or were added at the midpoint of cultivation.

Effect of addition of yeast extract We considered to separate the positive effects of the presence and activities of yeast 


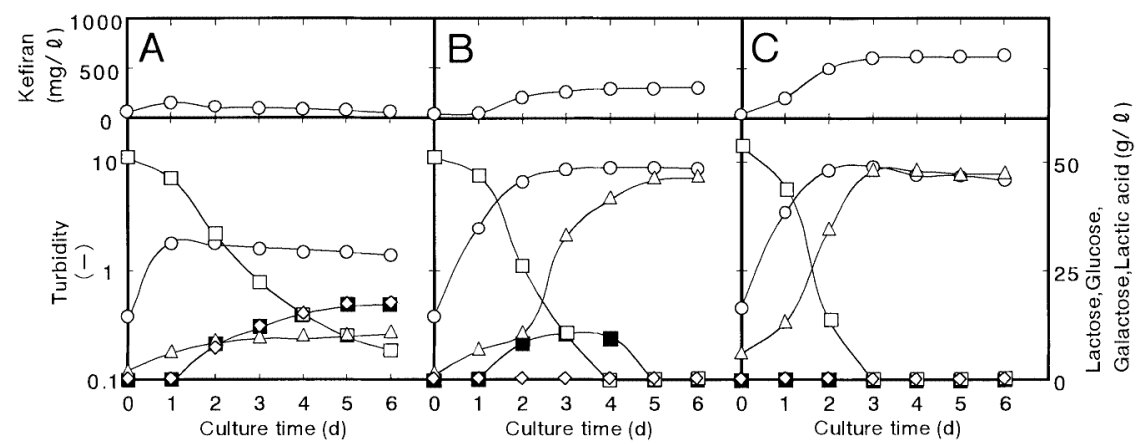

Fig 1. Effects of concentration of yeast extract initially added on kefiran production by L. kefiranofaciens. Yeast extract; A: 0 g/l, B: 2.5 g/l, C: 5 g/l. : Turbidity, $\square$ : Lactose, $\mathbf{n}$ : Galactose, $\diamond:$ Glucose, $\triangle$ : Lactic acid.

cells on kefiran production by L. kefiranofaciens JCM 6985 into factors of the supplementation of nutrients to the lactic acid bacteria and the provision of conditions suitable for cell growth and kefiran production by formation of $\mathrm{CO}_{2}$ and/or ethanol. As mentioned above, when the medium bottles were used, the $\mathrm{pH}$ of the culture broth dropped and consequently cell growth was inhibited by the accumulation of lactic acid. The concentration of kefiran produced in the cultivation without $\mathrm{pH}$ control using a bioreactor was around $460 \mathrm{mg} / \mathrm{l}$ using the modified MRS medium containing $50 \mathrm{~g} / \mathrm{l}$ of lactose and $5 \mathrm{~g} / \mathrm{l}$ of yeast extract. On the other hand, in other experiments using a bioreactor, cell growth and kefiran production were stimulated by controlling $\mathrm{pH}$ at 5.5 during cultivation as will be described below.

Figure 1 shows the effect of addition of yeast extract on kefiran production by L. kefiranofaciens JCM 6985. The $\mathrm{pH}$ of the culture broth was maintained at 5.5 throughout cultivation by adding $4 \mathrm{~N} \mathrm{NaOH}$ as described above. In the culture without yeast extract, poor growth was observed and hardly any kefiran was produced. The higher the concentrations of yeast extract added, the higher was the yield of kefiran. The addition of yeast extract at a concentration of $5 \mathrm{~g} / \mathrm{l}$ allowed a drastic increase in kefiran production. The final kefiran concentration was $650 \mathrm{mg} / \mathrm{l}$ in the culture with gentle agitation $(100 \mathrm{rpm})$, but without aeration. Yokoi et al. (1991) also pointed out that the essential nutrient for Lactobacillus sp. KPB-167B was yeast extract and that the lack of tryptone or meat extract achieved about $85 \%$ of the cell mass obtained in MRS medium. The enhanced cell growth

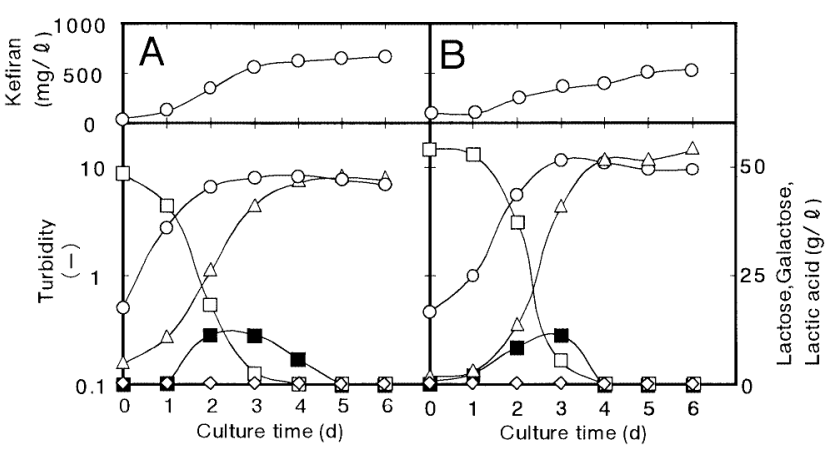

Fig 2. Effects of composition of a mixed gas for aeration on kefiran production by L. kefiranofaciens. A mixed gas of $\mathrm{N}_{2}$ and $\mathrm{CO}_{2}$ at a volume ratio of 9:1 (A) or 5:5 (B) was sparged at $0.3 \mathrm{vvm}$. Symbols are the same as shown in Fig. 1. and kefiran production appear to be mainly due to abundant vitamin groups in the yeast extract. It is necessary to examine the medium ingredients affecting the kefiran production; this point is especially important for the industrial production of kefiran.

Effect of aeration of $\mathrm{CO}_{2}$ Although L. kefiranofaciens JCM 6985 grew and produced kefiran in the cultivation without aeration as shown in Fig. 1C, no cell growth and no kefiran production by the bacterium were observed in the cultivation with aeration of $\mathrm{N}_{2}$ alone at $0.3 \mathrm{vvm}$ (data not shown). Then, a mixed gas of $\mathrm{N}_{2}$ and $\mathrm{CO}_{2}$ at a volume ratio of 9:1 or 5:5 was sparged into the culture broth at $0.3 \mathrm{vvm}$ throughout the cultivation. Figure 2 shows the effect of the composition of gas for aeration on kefiran production by L. kefiranofaciens JCM 6985. When a mixed gas of 9:1 $\left(\mathrm{N}_{2}: \mathrm{CO}_{2}\right)$ was sparged (Fig. 2A), a slight increase in the amount $(670 \mathrm{mg} / \mathrm{l})$ of kefiran produced was observed as compared with that $(650 \mathrm{mg} / l)$ in the cultivation without aeration shown in Fig. 1C. The supply of a slight amount of $\mathrm{CO}_{2}$ was found to be important for cell growth and kefiran production. Moreover, judging from the fact that galactose was detected once at the midpoint (2-4 days) of the cultivation, the aeration of gas containing $\mathrm{CO}_{2}$ caused a change in uptake and metabolism of lactose. However, the reason for the accumulation of galactose as an intermediate in the culture broth is still unclear.

Effect of addition of ethanol Prior to elucidation of the effect of ethanol addition on kefiran production, the effect of lactose concentration on the production by L. kefiranofaciens JCM 6985 was investigated in control cultivation without ethanol. Figure 3 shows the results of cultivations using a modified MRS

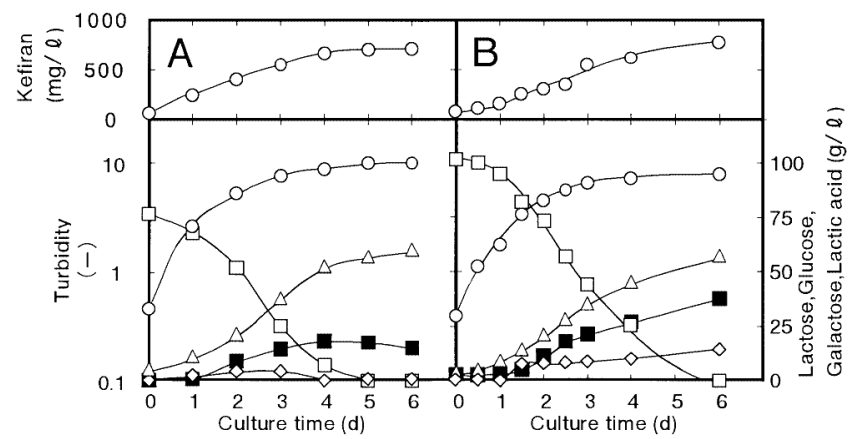

Fig 3. Production of kefiran by L. kefiranofaciens using the medium containing $75 \mathrm{~g} / \mathrm{l}(\mathrm{A})$ and $100 \mathrm{~g} / \mathrm{l}(\mathrm{B})$ of lactose as a carbon source. Symbols are the same as shown in Fig. 1. 


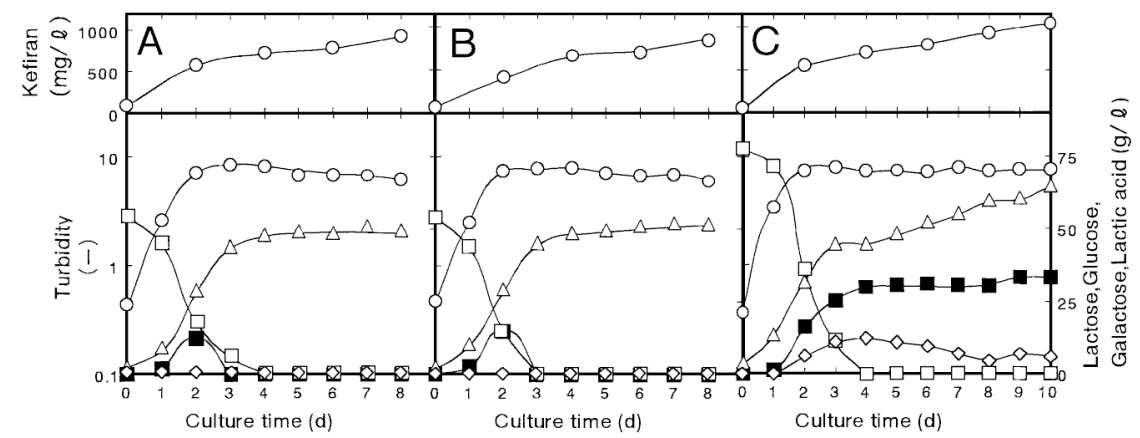

Fig 4. Effects of ethanol addition on kefiran production by L. kefiranofaciens. When the medium containing $50 \mathrm{~g} / \mathrm{l}$ of lactose was used as a carbon source, 10 $\mathrm{g} / l$ (A) or $20 \mathrm{~g} / l(\mathrm{~B})$ of ethanol was added. When the medium containing $75 \mathrm{~g} / l$ of lactose was used as a carbon source, $10 \mathrm{~g} / l$ (C) of ethanol was added. Symbols are the same as shown in Fig. 1.

medium containing 75 and $100 \mathrm{~g} / \mathrm{l}$ of lactose as a carbon source. A mixed gas of $\mathrm{N}_{2}$ and $\mathrm{CO}_{2}$ at a volume ratio of 9:1 was sparged into the culture broth at $0.3 \mathrm{vvm}$ throughout the cultivations. As shown in Fig.3A, $75 \mathrm{~g} / l$ of lactose was consumed completely by 5 days, but galactose accumulated gradually and remained in the culture broth at 6 days. Glucose accumulated once for 2-3 days and then was consumed completely by 4 days. The concentration of lactic acid gradually increased and reached a maximum value of $60.3 \mathrm{~g} / \mathrm{l}$ at 6 days. When $100 \mathrm{~g} / \mathrm{l}$ of lactose was used as a carbon source, it was consumed completely by 6 days, but glucose as well as galactose remained in the culture broth after 1 day as shown in Fig. 3B. In this cultivation, the final lactic acid concentration was $57.4 \mathrm{~g} / \mathrm{l}$. The amounts of kefiran produced from 75 and $100 \mathrm{~g} / \mathrm{l}$ of lactose were about $710 \mathrm{mg} / \mathrm{l}$ (Fig. 3A) and 770 $\mathrm{mg} / \mathrm{l}$ (Fig. 3B), respectively, which were higher than that $(670$ $\mathrm{mg} / l$ ) with the cultivation using $50 \mathrm{~g} / \mathrm{l}$ of lactose as a carbon source (see Fig. 2A). The yields of kefiran per gram of lactose added could not be enhanced by increasing the initial concentration of lactose. The results show that the accumulation of lactic acid at such a high level causes the inhibition of uptake and metabolism of lactose, just as with the aeration of gas containing $\mathrm{CO}_{2}$ described above. In addition, it is obvious that the accumulation of lactic acid leads to a decrease in growth rate as reported previously (Taniguchi et al., 1987, 1994; Burgos-Rubio et al., 2000). The removal of lactic acid from culture broth by separation methods such as membrane filtration, electrodialysis, and liquid-liquid extraction seems to allow not only achievement of high cell concentration but also enhancement of kefiran production. Research on the development of a culture system which removes lactic acid for kefiran production is currently under way.

The kefir made by the traditional procedure contains $0.5-1.5 \%$ of ethanol and a small amount of acetic acid as well as $0.6-1.0 \%$ of lactic acid. By adding initially 10 or $20 \mathrm{~g} / l$ of ethanol to the medium containing $50 \mathrm{~g} / \mathrm{l}$ or $75 \mathrm{~g} / \mathrm{l}$ of lactose, the effect of the addition on kefiran production by L. kefiranofaciens JCM 6985 was examined. Figure 4 shows the results of cultivations with the $\mathrm{pH}$ controlled at 5.5 and simultaneously with sparging of a mixed gas of $\mathrm{N}_{2}$ and $\mathrm{CO}_{2}(9: 1)$. When 10 or $20 \mathrm{~g} / \mathrm{l}$ of ethanol was initially added to the medium containing $50 \mathrm{~g} / \mathrm{l}$ of lactose, the time when galactose could be detected in the culture broth decreased as compared to that without ethanol, although negligible changes in the profiles of lactose consumption and lactic acid production were observed. As shown in Figs. 4A and 4B, kefiran continued to be produced gradually in the culture broth even after all sugars were consumed. The concentration of kefiran obtained when $10 \mathrm{~g} / \mathrm{l}$ or $20 \mathrm{~g} / \mathrm{l}$ of ethanol was added to the medium was about $930 \mathrm{mg} / l$ or $840 \mathrm{mg} / l$ at 8 days, respectively. These values were significantly higher than that $(670 \mathrm{mg} / l)$ of the cultivation without ethanol described above. When $10 \mathrm{~g} / \mathrm{l}$ of ethanol was added to the medium containing $75 \mathrm{~g} / \mathrm{l}$ of lactose, the maximum concentration of kefiran obtained was about 1040 $\mathrm{mg} / \mathrm{l}$ at 10 days as shown in Fig. 4C. However, in this cultivation, the added lactose was not utilized efficiently to produce kefiran because both $5.9 \mathrm{~g} / l$ of glucose and $33 \mathrm{~g} / l$ of galactose remained in the culture broth. The reduced sugar consumption seems to be due to the accumulation of more than $50 \mathrm{~g} / \mathrm{l}$ of lactic acid, in the same manner as the results of cultivation using $100 \mathrm{~g} / \mathrm{l}$ of lactose as a carbon source (see Fig. 3B).

Comparison of amounts of kefiran produced Figure 5 shows a comparison of the amounts of extracellular kefiran obtained under the different culture conditions. By sparging a mixed gas of $\mathrm{N}_{2}$ and $\mathrm{CO}_{2}$ at a volume ratio of 9:1 into the culture broth at $0.3 \mathrm{vvm}$ throughout the cultivation, a slight increase in the amount $(670 \mathrm{mg} / l)$ of kefiran produced was observed as compared with that $(650 \mathrm{mg} / l)$ in the cultivation without aeration. However, further increase in the $\mathrm{CO}_{2}$ fraction to one-half of total gas volume caused a decrease in the amount of kefiran produced. When $10 \mathrm{~g} / \mathrm{l}$ or $20 \mathrm{~g} / \mathrm{l}$ of ethanol was added to the medium containing $50 \mathrm{~g} / \mathrm{l}$ of lactose, the kefiran concentrations were about $930 \mathrm{mg} / \mathrm{l}$ or $840 \mathrm{mg} / \mathrm{l}$ at 8 days, respectively. The maximum kefi-

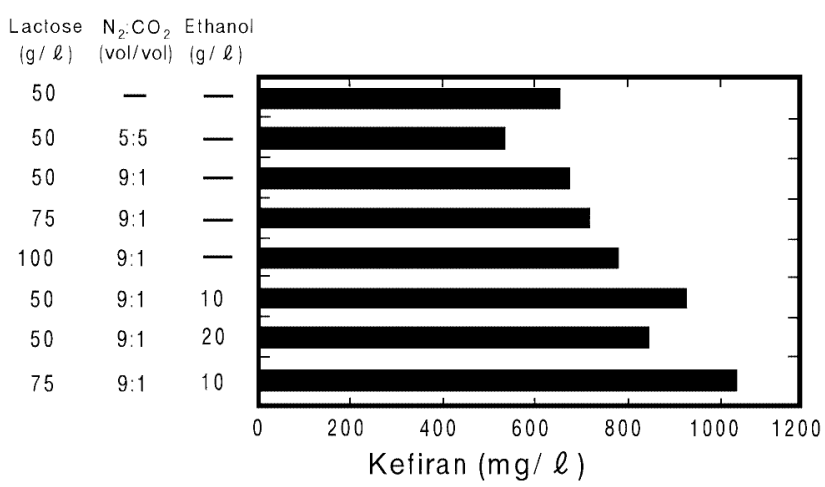

Fig 5. Comparison of the amounts of kefiran produced under different culture conditions. 
ran concentration was about $1040 \mathrm{mg} / \mathrm{l}$ at 10 days, when $10 \mathrm{~g} / \mathrm{l}$ of ethanol was added to the medium containing $75 \mathrm{~g} / \mathrm{l}$ of lactose. Although the time required to reach the maximum concentration of kefiran was longer, the addition of ethanol to the medium made it possible to increase the amount of kefiran produced as shown in Fig. 4.

Based on the results described above, we found that factors resulting from the presence of yeast strains in kefir grains, that is, addition of yeast extract and ethanol, aeration of gas containing $\mathrm{CO}_{2}$, and their combinations allowed promotion of kefiran production by L. kefiranofaciens. In general, to stimulate production of useful substances by cooperative actions between two microorganisms in a co-culture, it seems very difficult to optimize the culture conditions of $\mathrm{pH}$, temperature, composition of aeration gas, ratio of inoculum size, time and amount of inoculation of each microorganism, and composition of nutrients in the medium used. The results obtained in this study may show one good example of production of useful compounds using a single microorganism under culture conditions established by mimicking the actions of yeast cells on L. kefiranofaciens in kefir grain as a typically natural co-culture system.

Acknowledgements The authors wish to thank Dr. T. Toba, Department of Applied Bioscience, Hirosaki University, for helpful discussion. This work was supported in part by Grants-in-Aid for a special research project in the year 2000 from the MORINAGA HOSHI-KAI and for general research from the Cosmetology Research Foundation.

\section{References}

Crescenzi, V. (1995). Microbial polysaccharides of applied interest: ongoing research activities in Europe. Biotechnol. Prog., 11, 251259.

Burgos-Rubio, C.N., Okos, M.R. and Wankat, P.C. (2000). Kinetic study of the conversion of different substrates to lactic acid using Lactobacillus bulgaricus. Biotechnol. Prog., 16, 305-314.

Dubois, M., Gilles, K.A., Hamilton, J.K., Rebers, P.A. and Smith, F. (1956). Colorimetric method for determination of sugars and related substances. Anal. Chem., 28, 250-356.

Kooiman, P. (1968). The chemical structure of kefiran, the water-soluble polysaccharide of the kefir grains. Carbohydr. Res., 7, 200-211.

Micheli, L., Uccelletti, D., Palleschi, C. and Crescenzi, V. (1999). Isolation and characterisation of a ropy Lactobacillus strain producing the exopolysacchride kefiran. Appl. Microbiol. Biotechnol., 53, 6974.
Mitsue, T., Tachibana, K. and Fujio, Y. (1998). Isolation of kefiranproducing lactic acid bacteria from kefir grain and improvement of kefiran productivity. Seibutu-Kogaku Kaishi, 76, 447-450 (in Japanese).

Mitsue, T., Tachibana, K. and Fujio, Y. (1999). Efficient kefiran production by a mixed culture of Lactobacillus kefiranofaciens KF-75 and yeast strains. Seibutu-Kogaku Kaishi, 77, 99-103 (in Japanese).

Mukai, T., Toba, T., Itoh, T. and Adachi, S. (1990). Structural investigation of the capsular polysaccharide from Lactobacillus kefiranofaciens $\mathrm{K}_{1}$. Carbohydr. Res., 204, 227-232.

Mukai, T., Watanabe, N., Toba, T., Itoh, T. and Adachi, S. (1991). Gelforming characteristic and rheological properties of kefiran. J. Food Sci., 56, 1017-1026.

Shiomi, M., Sakai, K., Murofushi, M. and Aibara, K. (1982). Antitumor activity in mice of orally administered polysaccharide from kefir grain. Jpn. J. Med. Sci. Biol., 35, 75-80.

Tamime, A. and Robinson, R. (1988). Fermented milks and their future trends: Part II. Technological aspects. J. Dairy Res., 55, 281307.

Taniguchi, M., Kotani, N. and Kobayashi, T. (1987). High-concentration cultivation of lactic acid bacteria in fermentor with cross-flow filtration. J. Ferment. Technol., 65, 179-184.

Taniguchi, M., Hoshino, K., Shimizu, K., Nakagawa, I., Takahashi, Y. and Fujii, M. (1988). Rapid production of Pediococcus halophilus salt-tolerant cells by a cultivation method employing gradual increases in $\mathrm{NaCl}$ concentration using a fermentor with a microfiltration module. J. Ferment. Technol., 66, 633-641.

Taniguchi, M., Nakagawa, I., Hoshino, K., Itoh, T., Ohno, K. and Fujii, M. (1989). Production of superoxide dismutase from Streptococcus lactis using a bioreactor with a microfiltration module. Agric. Biol. Chem., 53, 2447-2453.

Taniguchi, M., Hoshino, K., Urasaki, H. and Fujii, M. (1994). Continuous production of an antibiotic polypeptide (nisin) by Lactococcus lactis using a bioreactor coupled to a microfiltration module. J. Ferment. Bioeng., 77, 704-708.

Toba, T., Abe, S., Arihara, K. and Adachi, S. (1986). A medium for the isolation of capsular bacteria from kefir grains. Agric. Biol. Chem., 50, 2673-2674.

Yokoi, H., Watanabe, T., Fujii, Y., Toba, T. and Adachi, S. (1990). Isolation of characterization of polysaccharide-producing bacteria from kefir grains. J. Dairy Sci., 73, 1684-1689.

Yokoi, H., Watanabe, T., Fujii, Y., Mukai, T., Toba, T. and Adachi, S. (1991). Some taxonomical characteristics of encapsulated Lactobacillus sp. KPB-167B isolated from kefir grains and characterization of its extracellular polysaccharide. Int. J. Food Microbiol., 13, 257264.

Yokoi, H. and Watanabe, T. (1992). Optimum culture conditions for production of kefiran by Lactobacillus sp. KPB-167B isolated from kefir grains. J. Ferment. Bioeng., 74, 327-329. 